Method. A scoping literature review was undertaken between January and March 2020. A single researcher searched OvidSP, psychinfo, relevant grey literature and undertook hand searches of key reference lists. Following PRISMA-SCR protocol, abstracts and articles were screened against inclusion/exclusion criteria to identify relevant papers. Papers were then subjected to critical appraisal and findings summarised using a narrative approach. Key data for blood pressure, pulse and body temperature were pooled and analysed in the context of wider findings.

Result. Data from 219 patients were included from 20 studies. 13 of these studies were case studies or case series, 5 were cross sectional and 2 were cohort studies. Cardiovascular compromise including bradycardia (61\%) and hypotension (30.3\%) were common and a single episode of cardiac arrest was documented in the literature. Bone density was reduced $(Z$ score $\leq 1)$ in $36.7 \%$ of cases. A wide variety of single episodes of physical morbidity were also documented including pneumothorax, liver dysfunction, growth retardation and thyroid dysfunction.

Conclusion. This scoping review highlights the physiological compromise experienced by some male adolescents with AN. Guidelines for the identification, assessment and management of physical health complications - including MARSIPAN by the Royal College of Psychiatrists - continues to use data heavily drawn from female-biased populations. Given the evidence summarised, there is concern that in the absence of specific guidance, adolescent males may be at high risk of negative outcomes including acute cardiovascular compromise, osteoporosis and reduced linear growth.

\section{An audit assessing the monitoring of SSRIS after initiation in children and adolescents}

\section{Ella McGowan \\ Black Country Healthcare}

doi: 10.1192/bjo.2021.159

Aims. To identify children and adolescents started on SSRIs to see if they are being followed up in accordance to NICE and Maudsley guidelines

Objectives

Has the patient been followed up after a week to check for adverse effects or improvement in their mental state?

Has the patient been re-evaluated every 4-6 weeks, if not is there an alternative plan?

If there is no improvement has the dose been increased?

If there is an adverse effect has the dose been lowered or the medication stopped?

Method. Paper case notes including clinic letters and handwritten notes were reviewed on the 19/10/2020. The following data were collected anonymously.

Age

Gender

Date seen / Date medication started

Name of medication

Date medication started

Date of Follow-up

Monitoring of improvement

Monitoring of adverse effects

Outcome of monitoring

Result. A total of 18 sets of cases were identified.

Follow-up occurred in 17 of the 18 cases.

The one case that had not been followed up had started the medication 8 weeks before the audit. The median follow-up time was 42 days (6 weeks). No cases were followed up within a week.

Monitoring of improvement was recorded in $88 \%$ of case notes reviewed.

Monitoring for adverse effects occurred in 36\% of case notes and none of these patients had reported any side effects. $53 \%$ of cases did not have monitoring of adverse effects documented. There were two patients $(11 \%)$ who did not take the medication as prescribed. One out of choice and one their parent had not collected it.

The medication dose was increased in $22 \%$ of patients without clear documentation of monitoring for adverse effects.

Conclusion. After discussion with the clinical lead it was decided it is impractical to follow up patients a week after starting medication. However, patients and their carers should be informed of the side effects and advised to contact CAMHS if adverse effects occur.

The area of practice that can be improved is the documentation of adverse effects at follow-up.

Recommendations:

All patients to be informed of the common side effects of the medication before it is initiated and advised to contact the CAMHS team if they have concerns

All CAMHS patients started on SSRIs should be followed up within 4-6 weeks

At follow-up any adverse events and clinical response should be discussed

An accurate record of the exchanges of the above information should be documented in the notes

Re-audit

Reward processing in autism spectrum disorder and psychopathy: a systematic review

Patrick McLaughlin*, Marija-Magdalena Petrinovic and Nigel Blackwood

IoPNN King's College London

${ }^{*}$ Corresponding author.

doi: 10.1192/bjo.2021.160

Aims. Emerging research suggests that aberrant reward processing may underpin much of the social dysfunction we see in psychiatric disorders. Two conditions associated with marked social dysfunction are Autism Spectrum Disorder (ASD) and Psychopathy. However, no review to date has directly contrasted reward processing in both conditions and incorporated literature on social and non-social rewards. This systematic review aims to: (i) identify and compare reward processing abnormalities in ASD and Psychopathy as demonstrated in task-based functional magnetic resonance imaging (fMRI) studies; and (ii) identify correlations between fMRI reward processing abnormalities and manifested symptoms, with a focus on those giving rise to social dysfunction. Method. The electronic databases PubMed, PsycINFO and EMBASE were searched to identify studies satisfying the following criteria: (i) a validated measure was used to assess ASD or Psychopathy; (ii) the study was published in an English language peer review journal; (iii) the age of participants was 18 years or older; (iv) individuals participated in a reward-based experimental paradigm; and (v) the response to the reward was measure using fMRI.

Result. A total of 12 articles were identified that satisfied inclusion criteria. Six studies examined reward processing in ASD and six studies examined reward processing in Psychopathy. All studies in both conditions indicated some degree of abnormal reward-related neural response. The most replicated findings were aberrant responses in the Ventral Striatum (VS). Autism 
Spectrum Disorder was typified by VS hypoactivation to social and non-social reward, while Psychopathy was associated with VS hyperactivation in response to non-social reward anticipation. No studies were identified of social reward in Psychopathy.

Conclusion. The reported fMRI findings correlate with clinical observations in both conditions. Reduced reward response in ASD to a range of social and non-social stimuli would provide a parsimonious account of the social and non-social deficits that characterise the condition. Enhanced responses to the anticipation of reward in Psychopathy provides an account of the ruthless and destructive pursuit of reward-driven behaviours not inhibited by immoral or aversive signals. If, as the literature suggests, reward circuitry dysfunction plays a role in the development and manifestation of symptoms in both conditions, reward processing and its underlying neural circuitry may represent important targets for the development of novel treatment strategies.

\section{The utility of the Brief Edinburgh Depression Scale (BEDS) in assessing severity of depression in advanced cancer patients}

\author{
Zeryab Meyer ${ }^{1 \star}$, Christopher Shiels ${ }^{2}$, Christopher Dowrick ${ }^{3}$ \\ and Mari Lloyd-Williams ${ }^{4}$ \\ ${ }^{1}$ School of Medicine, University of Liverpool; ${ }^{2}$ Research Associate, \\ Institute of Population Health Sciences, University of Liverpool; \\ ${ }^{3}$ Professor of Primary Medical Care, Institute of Population Health \\ Sciences, University of Liverpool and ${ }^{4}$ Professor and Honorary \\ Consultant in Palliative Medicine, Academic Palliative and Supportive \\ Care Studies Group (APSCSG), University of Liverpool \\ ${ }^{*}$ Corresponding author.
}

doi: 10.1192/bjo.2021.161

Aims. When using an assessment tool, brevity and validity are essential. Although brief depression inventories exist, they rely heavily on the inclusion of somatic symptoms. This can be problematic in advanced cancer populations; weight loss and sleep disturbance are for the most part ubiquitous in these patients and may not necessarily be indicative of depression.

The Brief Edinburgh Depression Scale (BEDS) is a 6-item shortened version of the Edinburgh Depression Scale which has been validated for use in patients with advanced cancer and is used internationally. The BEDS cut off threshold of 6/18 indicates that depression may be present. However, the BEDS currently provides no information regarding severity. The aim of this study is to establish severity thresholds for the BEDS by comparing it to another depression scale: the commonly used, rigorously validated, Patient Health Questionnaire (PHQ-9).

Method. 284 advanced cancer patients attending hospice day services in the North West of England completed both the PHQ-9 and the BEDS. Mean participant age was 66.7 (Standard Deviation $=13.2)$ and the sample contained both males $(\mathrm{n}=$ $102,36 \%)$ and females $(n=182,64 \%)$. BEDS severity thresholds with the highest Sensitivity (Sn) and Specificity (Sp) were selected based on their ability to predict PHQ-9 categories.

Result. A BEDS score of 4 to 6 was selected to indicate 'mild depression' $(\mathrm{Sn}=81.7, \mathrm{Sp}=65)$; 7 to 8 'moderate depression' $(\mathrm{Sn}=74.8, \mathrm{Sp}=78.7) ; 9$ to 11 'moderately severe depression' $(\mathrm{Sn}=82, \mathrm{Sp}=82.9)$ and 12 or more 'severe depression' $(\mathrm{Sn}=$ 63.2, $\mathrm{Sp}=92.8$ ). A linearly weighted kappa (with $\mathrm{s}$ weighting) showed a moderate level of agreement $(0.47,95 \%$ Confidence Interval: 0.40-0.54).

Conclusion. The BEDS is a simple and brief tool used to screen for depression in advanced cancer patients. It is administered throughout the UK and multiple translation studies have enabled its global a (including in resource poor countries). The severity thresholds calculated here are derived from a large sample of patients with advanced cancer attending hospice services and demonstrate acceptable sensitivity and specificity in relation to the PHQ-9, a thoroughly validated reference standard. We conclude that the generated BEDS thresholds support use of the BEDS in determining the presence and severity of depression in advanced cancer populations.

\section{Improving facilitation of ECT treatment for patients in an acute medical hospital}

\section{Vatsala Mishra*, Chun Chiang Sin Fai Lam, Marilia Calcia} and Isabel McMullen

Mental Health Liaison Team, King's College Hospital, South London and Maudsley NHS Foundation Trust

${ }^{*}$ Corresponding author.

doi: 10.1192/bjo.2021.162

Aims. A Quality Improvement Project aiming to streamline facilitation of electroconvulsive therapy (ECT) treatment for psychiatric patients at a general acute hospital and reduce cancellation rates via the use of a checklist.

ECT treatment is an essential aspect of psychiatric care for patients with severe depression or treatment-resistant psychosis. Facilitation of ECT treatment is an uncommon task for liaison psychiatry and the medical and nursing teams responsible for patients' medical care. Between August-October 2019, this liaison psychiatry team had 3 patients undergoing ECT treatment a total of 13 times, with treatment being cancelled on 4 occasions. After engagement with stakeholders from the acute medical teams, the liaison team and the ECT suite team, key areas requiring intervention were identified to help reduce the rates of cancellation. Areas identified included a lack of ownership on the logistic and operational aspects of ECT amongst staff, a lack of knowledge of what the process involved and a lack of confidence in managing said patients. Difficulties in communication between teams and accurate documentation may contribute to errors and cancellation of ECT sessions, which in turn would delay treatment and impact on patient safety and clinical outcomes.

Method. The first author, a Foundation Year 1 doctor, developed a 10-point checklist to be referred to when arranging ECT for patients, to ensure errors were not made which could lead to missed treatment and delayed recovery. The tasks and responsibilities of each key member of the team were clearly identified. This checklist was included in all ECT patients' files and teaching was provided to staff involved. Feedback was obtained from staff involved regarding the clarity of information and their confidence in managing such cases.

Result. In the month following initial intervention the liaison psychiatry team organised 12 ECT sessions. The checklist was pasted into notes the day before each ECT session and 0 sessions were missed for avoidable reasons. Feedback from staff showed all teams felt more confident co-ordinating ECT treatment as a result of the checklist.

Conclusion. Creating a 10-point checklist for the facilitation of ECT treatment in patients at a medical hospital was beneficial in reducing avoidable errors from $16 \%$ to $0 \%$. The liaison psychiatrists, medical doctors, and nurses involved reported greater confidence in managing patients undergoing ECT and described the checklist as enhancing the feeling of teamwork and communication within the multi-disciplinary team, and felt it had improved patient safety and clinical outcomes. 